CANCER

\title{
Treatment of colorectal and hepatocellular carcinomas by adenoviral mediated gene transfer of endostatin and angiostatin-like molecule in mice
}

\author{
V Schmitz, L Wang, M Barajas, C Gomar, J Prieto, C Qian
}

Gut 2004;53:561-567. doi: 10.1136/gut.2003.019307

See end of article for authors' affiliations .....................

Correspondence to: $\operatorname{Dr} C$ Qian/Dr V Schmitz, Division of Hepatology and Gene Therapy, Department of Medicine, Medical School, University of Navarra, 31080 Pamplona, Spain; CQIAN@UNAV.ES/ Volker.Schmitz@ ukb.uni-bonn.de

Accepted for publication 17 September 2003

\begin{abstract}
Aim and method: In this study, we explored the responsiveness of different tumour entities (colorectal carcinoma (CRC), hepatocellular carcinoma (HCC), and the murine Lewis lung carcinoma (LLC)) to angiostatic antitumour treatment with two recombinant adenoviral vectors encoding angiostatin-like molecule (AdK1-3) and endostatin (Adendo).

Results: AdK1-3 and Adendo exerted inhibitory biological functions on endothelial cell proliferation, migration, and tube formation in vitro. AdK1-3 inhibited significantly endothelial cell infiltration in vascular endothelial growth factor embedded Matrigel plugs in mice whereas Adendo showed only minor effects. Both AdK1-3 and Adendo induced similar antitumour effects in the LLC tumour model in immune competent $\mathrm{C} 57 \mathrm{BL} / 6$ mice but AdK1-3 had stronger inhibitory effects in athymic mice. Furthermore, AdK 1-3 inhibited tumour growth in a murine CRC and human HCC model but was ineffective in a human CRC model. In contrast, Adendo did not reduce tumour progress in either of these tumour models although AdK1-3 and Adendo effectively reduced intratumoral microvessel density in LLC tumours.

Conclusion: Our data demonstrate that angiostatic gene therapy may form a feasible strategy for the treatment of established hepatocellular carcinomas and that in vivo antitumour efficacy of angiostatic proteins is tumour specific.
\end{abstract}

$\mathrm{T}$ he role of angiogenesis in the development of neoplastic diseases has been unravelled in recent years. ${ }^{1-4}$ The underlying attractive rational for angiostatic gene transfer strategies is to let the body itself produce the antitumour drug. ${ }^{2}$ While most conventional antitumour strategies attack the malignant tumour cell, the main goal of angiostatic therapies is to prevent tumour progress by inhibiting tumour angiogenesis by high systemic levels of antiangiogenic proteins. ${ }^{356}$ Adenoviral vectors fulfil the basic requirements for angiostatic gene therapy by ensuring a high transduction efficacy allowing high circulating protein levels. Recombinant adenoviruses encoding angiostatin and endostatin have been shown in some studies to exert high antitumour and antimetastatic effects whereas other data were not as promising. ${ }^{7-12}$ A recent publication by Kuo et al showed for the first time in a comparative study design the responsiveness of different tumour entities towards different angiostatic compounds. ${ }^{7}$ This is a striking observation as any antiangiogenic antitumour therapy is thought to attack the tumour via genetically stable endothelial cells. Therefore, the antitumour responsiveness of different compounds could be expected to be similar when tested in different tumour entities. Taking this into account, further comparative studies are warranted to evaluate the role of angiostatic proteins in different tumour models.

In our study, two gastrointestinal tumour entities, hepatocellular carcinoma (HCC) and colorectal carcinoma (CRC), were included. They represent a logical aim for an angiostatic antitumour approach because they frequently show strong hypervascularisation. ${ }^{13-15}$ Recently, ex vivo gene transfer for angiostatin was shown to be effective in a murine model for HCC. ${ }^{16}$ To determine whether the antitumour effects of different angiostatic compounds vary in different experimental tumour models, we investigated the effects of angiostatin-like molecule and endostatin on tumour growth of established colorectal, hepatocellular, and lung carcinomas in mice.

\section{METHODS}

Animals and cell lines

C57BL/6, Balb/c, ror athymic mice, 5-8 weeks old, were purchased from Harlan (Barcelona, Spain). During the experimental period, mice were housed under pathogen free conditions. All animal procedures were performed according to approved protocols and in accordance with recommendations for proper care and use of laboratory animals.

The human and murine lung cancer cell lines A549 and Lewis lung carcinoma (LLC) cells, human HT-29 CRC cells, and 293 cells (embryonic El transformed kidney cell line) were obtained from American Type Culture Collection (ATCC, Rockville, Maryland, USA). Cells were cultured in Dulbecco's modified Eagle medium (DMEM) supplemented with $10 \%$ heat inactivated fetal bovine serum (FBS). Human umbilical vein endothelial (HUVE) cells were obtained from Cascade Biologics (Portland, Oregon, USA) and were cultured according to the supplier's instructions. The human HCC cell line Huh7 and murine colon cancer cells were cultured in DMEM supplemented with 10\% FBS.

\footnotetext{
Abbreviations: CRC, colorectal carcinoma; HCC, hepatocellular carcinoma; LLC, Lewis lung carcinoma; VEGF, vascular endothelial growth factor; DMEM, Dulbecco's modified Eagle medium; FBS, fetal bovine serum; HUVE, human umbilical vein endothelial; RT-PCR, reverse transcription-polymerase chain reaction; $\mathrm{CM}$, culture medium; $\mathrm{BrdU}$, bromodeoxyuridine; pfu, plaque forming units; $\mathrm{MOI}$, multiplicity of infection
} 
Construction of recombinant adenoviruses encoding angiostatin-like molecule and endostatin

Murine endostatin was generated by a reverse transcriptionpolymerase chain reaction (RT-PCR) technique using mouse liver RNA as template. Primers used for signal peptide of endostatin were 5'-GGATCCACCTCCAGGACCACAGGA-3' and $5^{\prime}$-GATATCATTCCCATCAGCGCTGGCAG-3'. Primers used for endostatin were 5'-GATATCCATACTCATCAGGA CTTTCA-3' and 5'-GGATCCAGAGGCCTATTTGGAGAAT-3' (underlined sequences indicate restriction enzyme sites for EcoRI and HamHI). PCR fragments for endostatin and endostatin signal peptide were cloned into cloning vector pCR2.1-TOPO (Invitrogen, Carlsbad, California, USA) to generate pCR2.1/S and pCR2.1/E. They were confirmed by DNA sequencing. Endostatin signal peptide was released by EcoRI/EcoRV from pCR2.1/S and closed into EcoRI/EcoRV sites of pCR2.1/E to generate pCR2.1/endo. The fused endostatin sequence was released by BamHI from pCR2.1/ endo and ligated into the BamHI site of pSQ1 resulting in pSQ1/endo. Angiostatin-like molecule Kl-3 was generated as described previously. ${ }^{29}$ Briefly, human plasminogen cDNA plasmid was used as template and the Kl-3 sequence was cloned into the cloning vector pCR2.1-TOPO (Invitrogen) to form pCR2.1/Kl-3. Kl-3 fragment was released by BamHI/ XhoI from pCR2.1/K1-3 and blunt end ligated into the BamHI site of pSQ1, resulting in pSQ1/Kl-3, and put under the control of the CMV immediate early promoter. Recombinant adenoviruses were generated as described previously. ${ }^{17}$ Briefly, pSQ1/Kl-3 and pSQ1/endo, respectively, were cotransfected with pJM17 into 293 cells by calcium phosphate precipitation. Recombinant adenoviruses were isolated from a single plaque, expanded, and purified by ultracentrifugation. Purified virus was dialysed against $10 \mathrm{mM}$ Tris/ $1 \mathrm{mM} \mathrm{MgCl}_{2}$ and stored in aliquots at $-80^{\circ} \mathrm{C}$. Virus concentration was determined by measuring virus particles and by cytotoxic plaque assay in 293 cells.

\section{Western blot}

Conditioned culture medium (CM $10 \mu \mathrm{l}$ ) of A549 cells which were infected with AdKl-3 or control vector AdlacZ for 70 hours was used for electrophoresis in $10 \%$ sodium dodecyl sulphate-polyacrylamide gel electrophoresis. Membranes were hybridised with a polyclonal antibody against plasminogen (Biogenesis, Pools, UK) and a secondary antibody against rabbit IgG (Sigma, St Louis, Missouri, USA) and were developed according to the protocol (ECL-Plus; Amersham Pharmacia Biotech, Little Chalfont, UK).

\section{ELISA}

Quantification of murine endostatin in supernatant from vector infected cells and mouse serum after receiving vectors was carried out using the Accucyte ELISA kit according to the manufacturer's instructions (Cytoimmune, College Park, Maryland, USA).

\section{Bromodeoxyuridine proliferation assay}

HUVE cells (5000) were resuspended in $40 \mu \mathrm{l}$ of CM and dispensed in each well of a 96 culture plate and preincubated with $60 \mu \mathrm{l}$ of CM. After a period of 30 minutes of preincubation, $100 \mu \mathrm{l}$ of HUVE cell CM containing growth supplement were added. Cell culture was continued for 42 hours and then cells were labelled with bromodeoxyuridine (BrdU) for a further six hours. The BrdU assay was performed according to the manufacturer's protocol (BrdU proliferation assay; Roche Diagnostics, Mannheim, Germany).

\section{Migration assay}

Inserts ( $8 \mu \mathrm{m}$ pores; Costar, Madrid, Spain) for 24 well culture plates were coated with $100 \mu \mathrm{g} / \mathrm{ml}$ of rat tail collagen type I (Becton Dickinson, Bedford, Massachusetts, USA). HUVE cells with a passage number of 4-6 (25000 cells/50 $\mu \mathrm{l}$ ) were seeded into the upper chamber. The lower chamber was filled with Medium 200 containing 1\% bovine serum albumin. HUVE cells were preincubated with $\mathrm{CM}$ at $37^{\circ} \mathrm{C}$ for 30 minutes before adding vascular endothelial growth factor (VEGF) at a final concentration of $5 \mathrm{ng} / \mathrm{ml}$ to the lower chamber. These chambers were incubated for six hours to allow cells to migrate through the collagen coated pore membranes. Non-migrated cells were thoroughly scraped off the upper surface membrane with cotton swabs. The membrane was stained with Diff-Quick (Dade Behring, Düdingen, Switzerland). Five to eight representative fields in each well were counted at $100 \times$ magnification to determine the number of migrated cells. Migration was expressed as percentage of maximal migration (defined as migration with VEGF stimulation without addition of CM).

\section{Tube formation assay}

The 24 well plate was coated with $320 \mu$ l Matrigel. HUVE cells $(25000$ cells/75 $\mu$ l) were dispensed into each well and incubated with $75 \mu \mathrm{l}$ of $\mathrm{CM}$ for 30 minutes. After adding $150 \mu \mathrm{l}$ of Medium 200 containing 10\% low serum growth supplement (Cascade Biologics, Portland, Oregon, USA), cells were incubated for another six hours. Tube formations were quantified by counting intact tubes in the whole well under microscopy at $40 \times$ magnification.

\section{In vivo testing of antiangiogenic effects (Matrigel angiogenesis assay)}

The Matrigel assay was performed with modifications, as described previously. ${ }^{25}$ Athymic mice were treated by intravenous injection of Adendo, AdKl-3, or AdlacZ at a dose of $10^{10}$ plaque forming units (pfu) per animal. Six hours later, $150 \mu \mathrm{l}$ of VEGF embedded Matrigel was injected subcutaneously into the left and right midabdominal region of mice. After 14 days mice were sacrificed and Matrigel plugs were removed and stained (haematoxylin-eosin). Quantitative analysis was done by counting the total number of endothelial-like cells in the Matrigel plugs with a graticule (ProSciTech, Queensland, Australia; surface patter $21 \mathrm{~mm}$ ) under a microscope. Results were expressed as mean (SEM) cell number per square scale $\left(\mathrm{mm}^{2}\right)$.

\section{In vivo testing of antitumoral effects}

For establishment of LLC in C57BL/6 and athymic mice, $10^{6}$ LLC cells were injected subcutaneously into the right flank of mice. When the tumours reached $50 \mathrm{~mm}^{3}$ in volume, animals were treated by systemic injection of AdKl-3, Adendo, or the control vector AdlacZ at a dose of $10^{10}$ pfu per animal. In the case of human HCC (Huh7) and human CRC (HT29), $2 \times 10^{6}$ and $10^{7}$ cells were injected subcutaneously into athymic mice, respectively. After 10 days, HCC bearing mice were treated by systemic injection of AdK1-3, Adendo, or control vector AdlacZ at a dose of $10^{10} \mathrm{pfu}$ per animal. Murine CRC were established by implantation of $5 \times 10^{5}$ CT-26 cells into the right hind of Balb/c mice. When murine (CT-26) or human (HT-29) CRC tumours reached approximately $50 \mathrm{~mm}^{3}$ in volume, tumour bearing mice were treated with intratumoral injection of AdK1-3, Adendo, or the control vector AdlacZ at a dose of $5 \times 10^{9}$ or $10^{10}$ pfu per animal, respectively. Tumour growth was monitored by caliper measurement. Tumour volume was calculated from the formula: $\mathrm{V}=$ length $\times$ width ${ }^{2} \times 0.52$. 


\section{Assessment of microvessel density}

Paraffin sections of tumours that received therapy with adenoviral vectors were immune stained with rabbit anti-von Willebrand factor (factor VIII-related antigen; Dako, Glostrun, Denmark) and followed by sequential incubation of the sections with LSAB2 system (Dako). Enzymatic activity was developed using DAB (Dako) as chromogenic substrate and followed by counterstaining with Mayer haematoxylin.

\section{Statistical analysis}

Tumour data are given as mean (SEM) tumour volume. Differences between tumour volumes in different experimental groups were analysed for statistical significance by a non-parametric two tailed test (Mann-Whitney test) for unpaired samples; in the case of histology sections, differences between groups were calculated by the Student's $t$ test for unpaired samples. A p value of $<0.05$ was considered significant.

\section{RESULTS}

Construction of adenoviruses containing angiostatinlike molecule and endostatin (AdK 1-3 and Adendo)

Recombinant adenoviral vectors coding for angiostatin-like molecule and endostatin were constructed. The cDNA sequence was confirmed by sequencing, and transgene expression was shown in A549 cells infected with different multiplicities of infection (MOIs) of AdK1-3 and Adendo by RT-PCR. The predicted PCR products were detected in the AdK1-3 and Adendo groups whereas no band was amplified in cells infected with the control vector AdlacZ. Expression of the housekeeping gene, $\beta$-actin, was similar in all samples (results not shown). In order to demonstrate whether transgene products can be secreted into the supernatant of A549 cells infected with AdKl-3 and Adendo, supernatant from infected cells was collected for detection of angiostatinlike molecule by western blot and detection of endostatin by ELISA. Figure 1A shows that angiostatin was detected at a molecular weight of $38 \mathrm{kDa}$ only in supernatant from AdKl-3 infected cells and not in supernatant from control vector AdlacZ infected cells. We also found that secretion of endostatin into the cell supernatant of Adendo infected A549 cells was dose dependent, with the highest level of approximately $900 \mathrm{ng} / \mathrm{ml}$ at an MOI of 500 (fig 1B). These data demonstrate that infection with AdKl-3 and Adendo induces corresponding protein expression and secretion in infected tumour cells.

To demonstrate in vivo production of endostatin by adenoviral vectors, Balb/c mice that received Adendo or control vector AdlacZ at a dose of $10^{10}$ pfu per animal or saline intravenously were sacrificed seven days later and blood samples collected for measurement of endostatin. Figure IC shows that high levels of endostatin were found in animals that had been receiving Adendo but not in those with AdlacZ or saline application.

\section{Biological activity of angiostatin-like molecule and} endostatin produced by AdK 1-3 and Adendo in vitro Angiostatic proteins can be characterised by inhibitory effects on different endothelial cell functions: proliferation, migration, and tube formation. To evaluate the antiproliferative effect of CM from A549 cells infected with AdK1-3, Adendo, or control vector AdlacZ, HUVE cells were preincubated with $\mathrm{CM}$ at a final concentration of $30 \%$. We discovered that preincubation of HUVE cells with CM from AdKl-3 and Adendo infected cells resulted in inhibition of proliferation by $39 \%$ and $38 \%$, respectively, in comparison with AdlacZ (fig 2A). Both proteins also showed similar inhibitory effects on tube formation of HUVE cells. Tube formation was reduced by approximately $43 \%$ and $39 \%$, respectively,
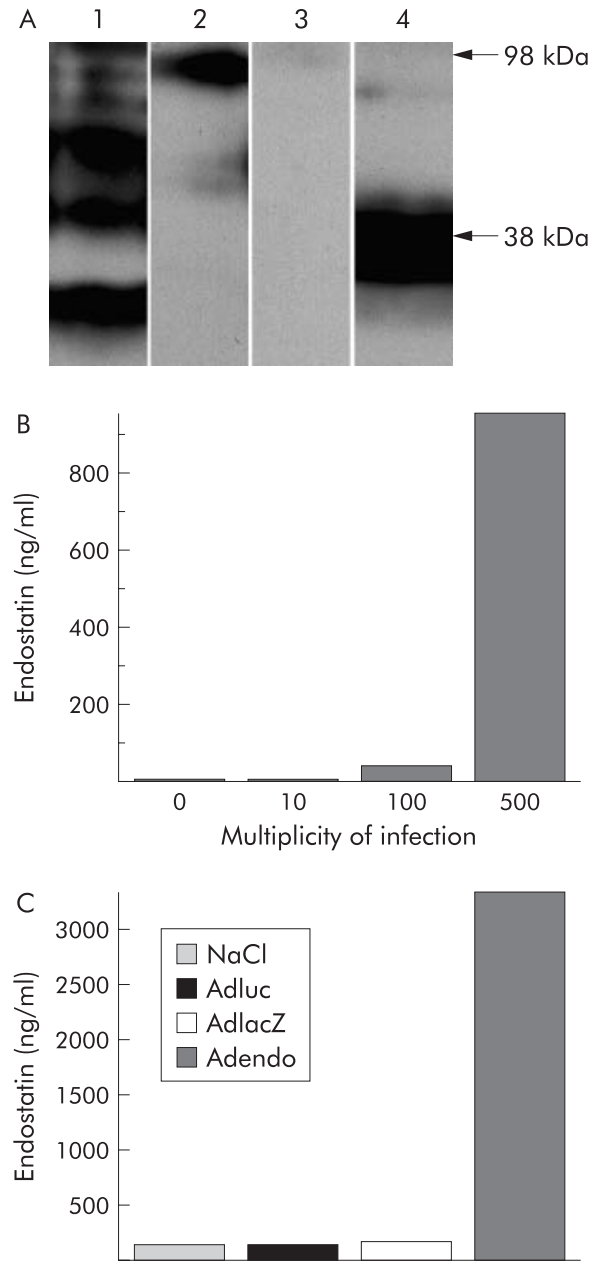

Figure 1 Western blot analysis for angiostatin (A) and ELISA for endostatin (B). Culture medium (CM) from uninfected and vector infected cells was subjected to western blot and ELISA, as described in the methods section. Lane 1, marker; lane 2, CM from cells infected with AdK 1-3; lane 3, recombinant plasminogen. Angiostatin fragments were detected at $38 \mathrm{kDa}$ in CM from AdK1-3. Endostatin levels are presented after infection of A549 cells at different multiplicities of infection. (B) In vitro data and $(C)$ in vivo data are shown, seven days after systemic administration of Adendo $\left(10^{10}\right.$ plaque forming units/mouse; pooled serum of two Balb/c mice).

compared with the AdlacZ control (fig 2B). While addition of CM to AdlacZ infected A549 cells did not affect the ability of HUVE cells to migrate through a perforated membrane, addition of CM containing $\mathrm{Kl}-3$ and endostatin inhibited cell migration by $64 \%$ and $86 \%$, respectively (fig 1C).

\section{Inhibition of angiogenesis in vivo by $\mathrm{AdK} 1-3$ and Adendo}

To evaluate whether angiostatin-like molecule and endostatin produced by AdKl-3 and Adendo inhibited angiogenesis in vivo, animals were inoculated subcutaneously with VEGF containing Matrigel, six hours after systemic administration of AdKl-3, Adendo, or the control vector AdlacZ. Matrigel plugs were collected for further histological examination two weeks later. As shown in fig 3, intense invasion by endothelium-like cells was observed in Matrigel plugs from animals that had received saline and control vectors AdlacZ. In contrast, Matrigel plugs from mice that had received AdK1-3 showed much less infiltration of endothelium-like cells. Administration of Adendo induced only minor inhibitory effects compared with control animals who received 

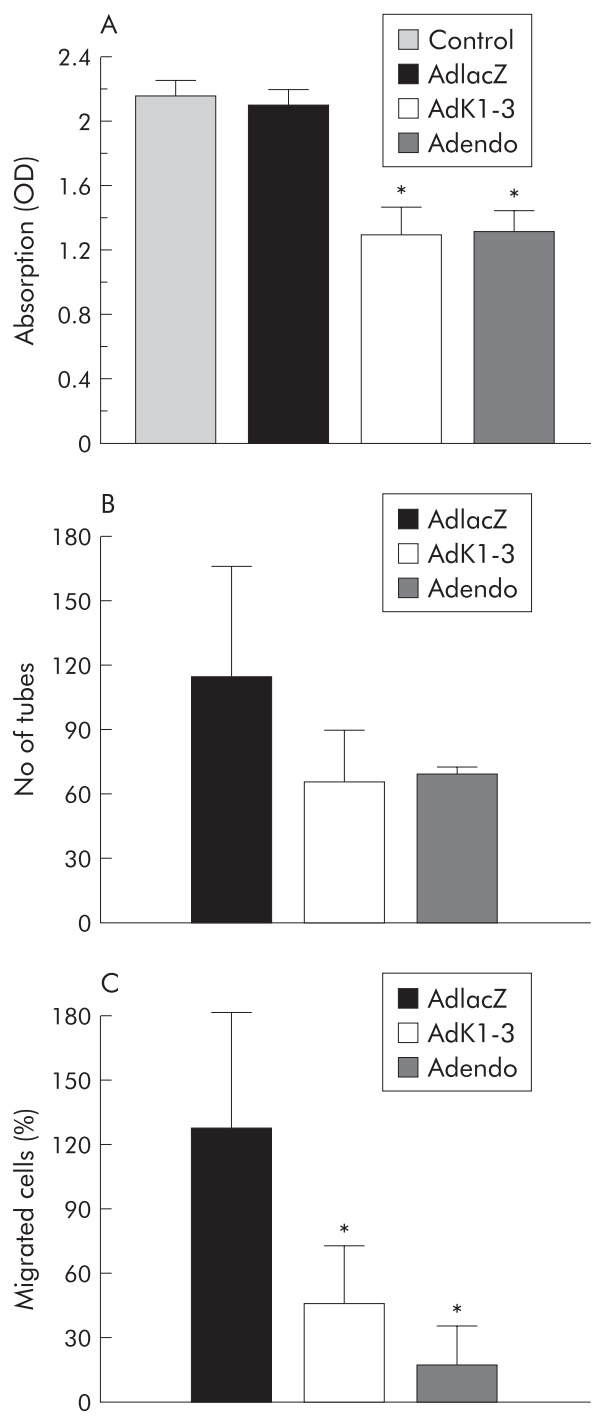

Figure 2 Inhibition of human umbilical vein endothelial (HUVE) cell proliferation (A), migration (B), and tube formation (C) by angiostatinlike molecule and endostatin expressed by adenoviral vectors. Data are given as mean (SEM) of 4-8 independent experiments. ${ }^{*} \mathrm{p}<0.05$ (AdK 1-3 and Adendo) compared with controls (control and Adluc). (B) Inhibition of HUVE cell tube formation by angiostatin expressed by adenoviral vectors. HUVE cells were preincubated with culture medium for 30 minutes in Matrigel coated culture wells and grown in culture medium supplemented with growth factor for an additional six hours. At this time point, HUVE cells started to form capillary-like structures. Intact tubes were quantified in the whole well under a microscope. Data are given as mean (SEM) of two independent experiments compared with control vector AdlacZ.

saline or AdlacZ. Quantification of endothelium-like cells on Matrigel sections (fig 3) showed that administration of AdKl-3 and Adendo resulted in a reduction in cell infiltration of $48 \%$ and $13 \%$, respectively, in comparison with animals who received the control vector Adlacz.

\section{Effect of systemic administration of AdK 1-3 and Adendo on established LLC tumours in C57BL/ 6 and athymic mice}

The antitumoral effect of AdKl-3 and Adendo was firstly evaluated in subcutaneous LLC tumours in C57BL/6 mice. When tumour sizes reached approximately $50 \mathrm{~mm}^{3}$ in volume, animals were treated by intravenous administration

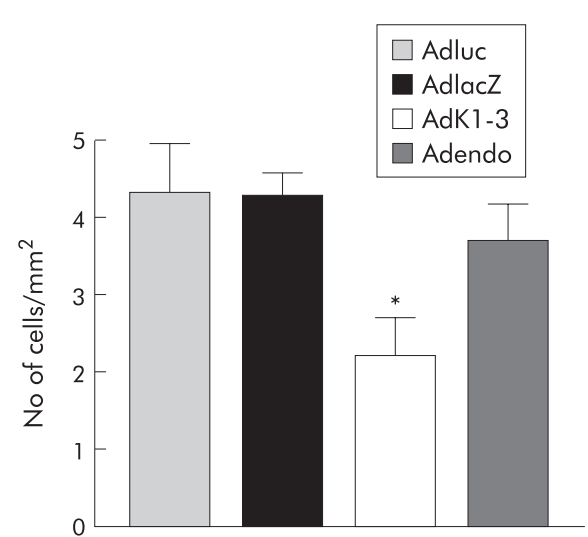

Figure 3 Suppression of vascular endothelial growth factor (VEGF) induced angiogenesis in vivo by systemic administration of Adendo and AdK1-3. Matrigel containing VEGF was injected subcutaneously into mice that had received intravenous administration of different adenoviruses six hours previously. After 14 days, Matrigel plugs were removed and stained with haematoxylin-eosin. Quantitative analysis of angiogenesis was made by counting the number of endothelium-like cells in an area $\left(\mathrm{mm}^{2}\right)$. Data are expressed as mean (SEM), $n=4 .{ }^{*} p<0.05$ compared with control animals who received AdlacZ or saline (unpaired Student's $t$ test).

of AdK1-3, Adendo, or AdlacZ at a dose of $10^{10} \mathrm{pfu} / \mathrm{animal}$. Figure 4A shows that treatment with AdK1-3 or Adendo resulted in a significant reduction in tumour progress by $54 \%$ and $46 \%$ four days after treatment initiation, respectively, in comparison with Adlacz. Analogous experiments were performed in athymic mice bearing established LLC tumours. As shown in fig 4B, treatment with AdK1-3 induced only slight inhibition of tumour growth, and treatment with Adendo was ineffective.

\section{Effect of systemic administration of AdK 1-3 and Adendo on established HCC tumours in athymic mice} HCC are a rational target for angiostatic antitumoral gene therapy because they frequently show hypervascularisation. Hence we administered AdK1-3 and Adendo to athymic mice bearing subcutaneous human Huh7 HCC tumours. Ten days later, animals were treated by intravenous administration of AdK1-3, Adendo, or AdlacZ at a dose of $1 \times 10^{10} \mathrm{pfu} / \mathrm{animal}$. As shown in fig 5, tumour growth was significantly inhibited by systemic administration of AdK1-3. Treatment with Adendo resulted in only slight inhibition of tumour growth. On day 7 after treatment, the inhibition rate induced by AdKl-3 or Adendo reached $87 \%$ and $46 \%$, respectively, compared with control animals.

\section{Effect of AdK 1-3 and Adendo on established subcutaneous colorectal cancer, CT-26 and HT-29, in Balb/c and athymic mice}

To evaluate the antitumoral effect of AdKl-3 and Adendo on CRC, murine CT-26 and human HT-29 CRC cells were inoculated subcutaneously into Balb/c or athymic mice, respectively. When CT-26 tumours reached a size of approximately $50 \mathrm{~mm}^{3}$, tumour treatment was initiated by intratumoral injection of the corresponding vectors at a concentration of $5 \times 10^{9} \mathrm{pfu} / \mathrm{mouse}$. Again, AdK1-3 induced a significant reduction in tumour growth by up to $41 \%$ on day 8 compared with the saline control whereas Adendo had no inhibitory effect (fig 6). Subcutaneous HT-29 tumours were treated by intravenous $(n=5$ each group) or intratumoral ( $\mathrm{n}=9$ each group) injection of AdKl-3, Adendo, or AdlacZ, respectively, at a concentration of $10^{10} \mathrm{pfu} / \mathrm{mouse}$, 

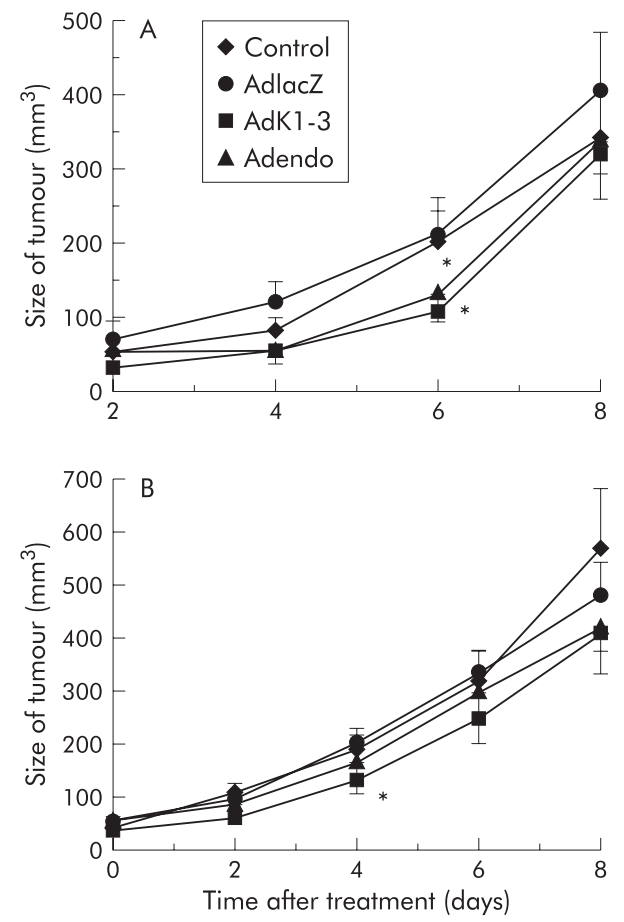

Figure 4 Inhibition of tumour growth on established Lewis lung carcinoma (LLC) tumours in C57BL/6 and athymic mice by systemic administration of AdK1-3 or Adendo. Established LLC tumours of approximately $50 \mathrm{~mm}^{3}$ in C57BL/6 mice (A) and athymic mice (B) were treated by intravenous injection of recombinant adenoviruses $\left(10^{10}\right.$ plaque forming units/mouse) or the control vector AdlacZ or saline. Tumour sizes were measured and are presented as mean (SEM), $n=7-13 .{ }^{*} p<0.05$ for AdK 1-3 and Adendo (A) and for AdK1-3 (B) compared with the AdlacZ control (day 4, Mann-Whitney).

respectively. Mean tumour volumes reached $189 \mathrm{~mm}^{3}$ and $291 \mathrm{~mm}^{3}$, respectively, compared with $183 \mathrm{~mm}^{3}$ in the AdlacZ group eight days after intratumoral vector administration (data not shown). Neither Adendo nor AdKl-3 inhibited tumour growth in this model in comparison with the AdlacZ control.

\section{Inhibition of tumour angiogenesis by systemic administration of AdK 1-3 and Adendo in a subcutaneous LLC model}

The underlying antitumoral mechanisms could be attributed, at least in part, to angiostatic effects of angiostatin-like molecule and endostatin as intratumoral vessel density was decreased significantly in both treatment groups compared with the AdlacZ control. For this purpose, intratumoral microvessels were immune stained against von Willebrand factor in LLC tumours. LLC tumours were removed seven days after treatment initiation. Tumours from control animals, Adlac $\mathrm{Z}$ and $\mathrm{NaCl}$, showed intensive staining against von Willebrand factor, indicating extensive angiogenesis in these tumours. In contrast, tumour samples from Adendo or AdKl-3 treated animals showed significant reduction of microvessel density of approximately $60 \%$. (fig 7 )

\section{DISCUSSION}

In this study, we showed that generation of angiostatin-like molecule (representing the first three kringles (Kl-3) of plasminogen) and endostatin by adenoviral mediated gene transfer caused tumour and protein specific antitumoral effects in three different tumour entities in mice: colorectal, hepatocellular, and Lewis lung carcinoma. Tumour and

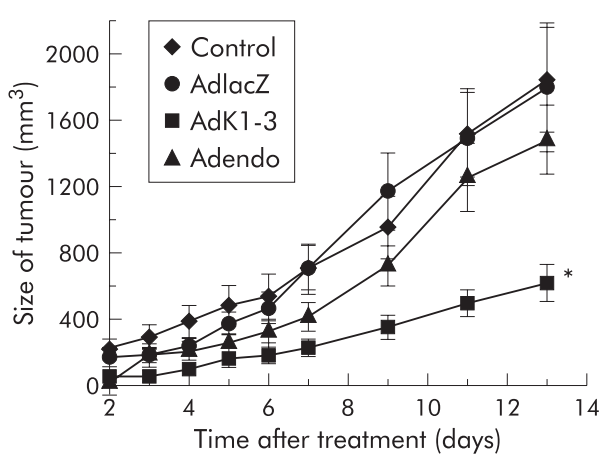

Figure 5 Inhibition of tumour growth in athymic mice with hepatocellular carcinoma tumours by systemic administration of AdK 1-3 or Adendo. Ten days after implantation of Huh7 cells, mice were treated by intravenous injection of recombinant adenoviruses $\left(10^{10}\right.$ plaque forming units/mouse) or saline as control. Size of tumour was measured and presented as mean (SEM), $n=6-10 .{ }^{*} \mathrm{p}<0.05$ for AdK $1-3$ day 3 -day 13 compared with the control groups who received AdlacZ (Mann-Whitney).

protein specificity of angiostatic tumour therapies is a striking aspect as tumour angiogenesis is considered to be a common pathological process independent of tumour type. Our findings indicate that different tumour entities have to be specifically evaluated for antitumour responsiveness towards distinct angiostatic proteins.

Recently, antiangiogenic tumour therapy attracted broad attention, and experimental data showed promising results with complete tumour elimination in some cases. ${ }^{18} 19$ The underlying concept appears convincing: to cut off the blood supply to the tumour and hence starve the tumour. ${ }^{20}$ The two physiological proteins, angiostatin and endostatin, showed antitumoral effects in several studies. ${ }^{78^{10} 18-25}$ However, production of the corresponding recombinant proteins is complicated..$^{24-28}$ It has been shown recently that ex vivo gene transfer for angiostatin (representing the first four kringles of plasminogen) reduced effectively the tumorigenicity of HCC cells. ${ }^{16}$ However, in vivo treatment of established tumours remains challenging. Adenoviral mediated gene transfer for cDNA encoding angiostatic proteins is a promising approach allowing the body to produce its own antitumour drug. Indeed, several experimental studies, including our own, demonstrated that recombinant adenoviruses are an appropriate vehicle for gene transfer of angiostatin(-like molecule) and endostatin. ${ }^{810-12} 29$ Sauter

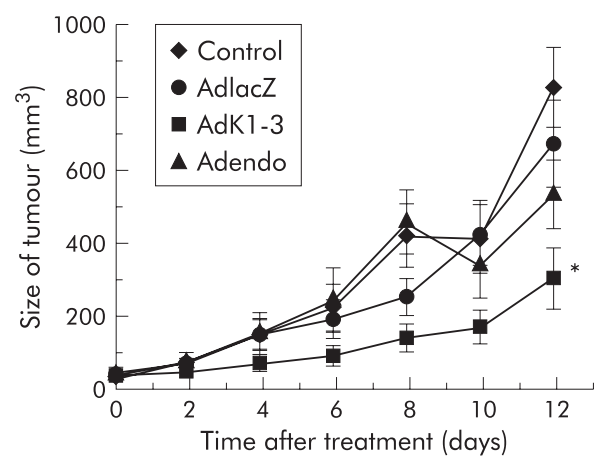

Figure 6 Inhibition of tumour growth on established colorectal cancer (CRC) tumours by systemic administration of AdK1-3 or Adendo. Intratumoral vector administration $\left(5 \times 10^{9}\right.$ plaque forming units/mouse) was initiated when tumours reached approximately $5 \mathrm{~mm}$ in mean diameter. Data are presented as mean (SEM), $n=7-11$. " $p<0.05$ for AdK1-3 day 4-day 12 compared with the AdlacZ control (MannWhitney). 


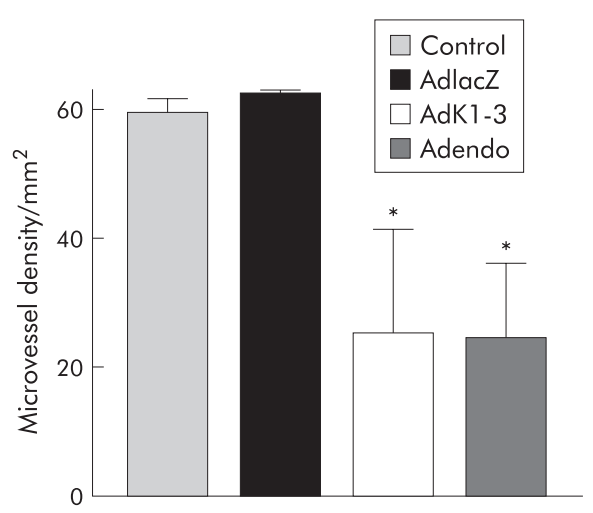

Figure 7 Inhibition of tumour angiogenesis by treatment with AdK 1-3 or Adendo. Established Lewis lung carcinoma tumours of approximately $50 \mathrm{~mm}^{3}$ in athymic mice were treated by intravenous injection of recombinant adenoviruses ( $10^{10}$ plaque forming units/mouse) or saline as control. Animals were sacrificed on day 7 after treatment and tumour tissue was removed. Microvessel density was determined by anti-von Willebrand factor staining. Quantitative analysis of microvessel density was made by counting the positively stained cells in 10 high power fields (HPF, $400 \times$ magnification). Data are given as mean (EM) cell number/ HPF, $n=3 .{ }^{*} \mathrm{p}<0.05$ for Adendo compared with AdlacZ (Student's $t$ test).

et al found strong antimetastatic effects of endostatin by gene transfer but only minor effects on primary tumour growth. ${ }^{10}$ In a comparative antitumour study, angiostatin and endostatin were less effective than VEGF receptor antagonists when tested in T241 fibrosarcomas. ${ }^{7}$

To our knowledge, the latter publication was the first to focus on a direct comparison of different angiostatic gene therapies. Our study focused on gene delivery for angiostatinlike molecule and endostatin, and confirmed the reported antitumoral effects in subcutaneous LLC tumours in immune competent mice. In addition, we observed that only angiostatin-like molecule maintained significant antitumoral effects in immune deficient mice. Furthermore, we showed for the first time in a direct comparative study that administration of AdKl-3 resulted in significant antitumoral effects in a human Huh7 HCC and a murine CT-26 CRC model whereas no effects of AdKl-3 were detected in a human HT-29 CRC tumour model. Despite similar in vitro effects on endothelial cell functions and in vivo effects in the LLC tumour model, endostatin had no effect in these particular tumour models. Several mechanisms may be involved in these tumour specific antitumour efficicacies: (1) protein expression and protein levels may be mouse strain specific and thereby influence antitumour effects; (2) angiostatic antitumour effects may involve primary or secondary immune responses dependent on the applied protein; and (3) tumour angiogenesis is a tumour specific process.

Wen et al showed large discrepancies between time periods and serum levels of protein expression in different mouse strains after gene delivery of endostatin. ${ }^{11}$ Although this may contribute to our findings, it is probably not the sole reason as we documented antitumoral effects over a short time period of only two weeks. Interestingly, the authors found strong antimetastatic effects for endostatin in an EOAMA metastasis model in 129/J mice but there was no effect for B16 melanoma in a metastasis model in C57BL6/J mice. However, in our study, treatment of LLC tumours by adenoviral mediated gene transfer for angiostatin and endostatin was effective in the C57BL6/J mouse strain. Our findings correspond well to the antitumour and antimetastatic effects found for LLC tumours described by Sauter and colleagues. ${ }^{10}$ Thus it can be concluded that the antitumoral effects of endostatin do not depend on the mouse strain but on the tumour entity that is treated, indicating that tumour angiogenesis may have to be reconsidered as a complex tumour specific process.

Considering the different antitumour effects for LLC tumours in immune competent and immune deficient mice, secondary or primary immune responses contribute essentially to the antitumoral effects of endostatin but are less important for the antitumour efficacy of angiostatin-like molecule. This is supported by our observation that reduction of microvessel density in LLC tumours was similar for endostatin and angiostatin-like molecule.

Taking into account the previous studies of Kuo and colleagues $^{7}$ and Wen and colleagues, ${ }^{11}$ our data further strengthen the hypothesis that tumour vascularisation is a tumour specific process and therefore requires the use of different antitumoral angiostatic proteins. As this aspect is complicating angiostatic antitumour therapy, further studies focusing on basic molecular mechanisms of tumour angiogenesis are warranted. Nevertheless, this study also underlines the fact that angiostatin-like molecule has significant antitumour effects in a human hepatocellular carcinoma model and therefore may contribute towards alternative therapy strategies for this tumour entity.

\section{ACKNOWLEDGEMENTS}

This work was supported in part by SAF 98-0146 from CICYT and also by J Vidal, MJ Huarte, Dr Cervera, M Losantos, and M Mendez grants for Gene Therapy. VS was partly supported by the German Exchange Council (DAAD) and the VERUM Foundation, Munich, Germany. We thank Helena Villanueva and Ingeborg Höschler for their expert assistance.

\section{Authors' affiliations}

V Schmitz*, L Wang, M Barajas, C Gomar, J Prieto, C Qian, Division of Hepatology and Gene Therapy, Department of Medicine, Medical School, University of Navarra, Pamplona, Spain

Present address: *Department of Internal Medicine, University Hospital Bonn, Germany

\section{REFERENCES}

1 Ellis LM, Liu W, Ahmad SA, et al. Overview of angiogenesis: Biologic implications for antiangiogenic therapy. Semin Oncol 2001;28(suppl 16):94-104.

2 Cao Y. Endogenous angiogenesis inhibitors and their therapeutic implications. Int J Biochem Cell Biol 2001;33:357-69.

3 Gasparini G. The rationale and future potential of angiogenesis inhibitors in neoplasia. Drugs 1999;58:17-38.

4 Soff GA. Angiostatin and angiostatin-related proteins. Cancer Metastasis Rev 2000;19:97-107.

5 Griffioen AW, Molema G. Angiogenesis: potentials for pharmacologic intervention in the treatment of cancer, cardiovascular diseases, and chronic inflammation. Pharmacol Rev 2000;52:237-68.

6 Pepper MS. Extracellular proteolysis and angiogenesis. Thromb Haemost 2001;86:346-55.

7 Kuo CJ, Farnebo F, Yu EY, et al. Comparative evaluation of the antitumor activity of antiangiogenic proteins delivered by gene transfer. Proc Natl Acad Sci U S A 2001;98:4605-10.

8 Griscelli F, Li H, Bennaceur-Griscelli A, et al. Angiostatin gene transfer: inhibition of tumor growth in vivo by blockage of endothelial cell proliferation associated with a mitosis arrest. Proc Natl Acad Sci U S A 1998;95:6367-72.

9 Griscelli F, Li H, Cheong C, et al. Combined effects of radiotherapy and angiostatin gene therapy in glioma tumor model. Proc Natl Acad Sci U S A 2000;97:6698-703.

10 Sauter BV, Martinet O, Zhang WJ, et al. Adenovirus-mediated gene transfer of endostatin in vivo results in high level of transgene expression and inhibition of tumor growth and metastases. Proc Natl Acad Sci U S A 2000;97:4802-7.

11 Wen XY, Bai Y, Stewart AK. Adenovirus-mediated human endostatin gene delivery demonstrates strain-specific antitumor activity and acute dosedependent toxicity in mice. Hum Gene Ther 2001;12:347-58.

12 Feldman AL, Restifo NP, Alexander HR, et al. Antiangiogenic gene therapy of cancer utilizing a recombinant adenovirus to elevate systemic endostatin levels in mice. Cancer Res 2000;60:1503-6.

13 Nakashima Y, Nakashima O, Hsia CC, et al. Vascularization of small hepatocellular carcinomas: correlation with differentiation. Liver 1999;19:12-18. 
14 Okamoto K, Oshika $Y$, Fukushima $Y$, et al. Inhibition of liver metastasis of colon cancer by in vivo administration of anti-vascular endothelial growth factor antibody. Oncol Rep 1999;6:553-6.

15 Ogawa H, Sato Y, Kondo M, et al. Combined treatment with TNP-470 and 5fluorouracil effectively inhibits growth of murine colon cancer cells in vitro and liver metastasis in vivo. Oncol Rep 2000;7:467-72.

16 Ishikawa H, Nakao K, Matsumoto K, et al. Antiangiogenic gene therapy for hepatocellular carcinoma using angiostatin gene. Hepatology 2003:37:696-704.

17 Qian C, Bilbao R, Bruna O, et al. Induction of sensitivity to ganciclovir in human hepatocellular carcinoma cells by adenovirus-mediated gene transfer of herpes simplex virus thymidine kinase. Hepatology 1995;22:118-23.

18 O'Reilly MS, Holmgren L, Chen C, et al. Angiostatin induces and sustains dormancy of human primary tumors in mice. Nat Med 1996;2:689-92.

19 O'Reilly MS, Boehm T, Shing Y, et al. Endostatin: an endogenous inhibitor of angiogenesis and tumor growth. Cell 1997;88:277-85.

20 Folkman J. Angiogenesis in cancer, vascular, rheumatoid and other disease. Nat Med 1995; 1:27-31.

21 Cao Y, Ji RW, Davidson D, et al. Kringle domains of human angiostatin. Characterization of the anti-proliferative activity on endothelial cells. J Biol Chem 1996;271:29461-7.
22 Cao Y Chen A, An SS, et al. Kringle 5 of plasminogen is a novel inhibitor of endothelial cell growth. J Biol Chem 1997;272:22924-8.

23 Cao R, Wu HL, Veitonmaki N, et al. Suppression of angiogenesis and tumor growth by the inhibitor K1-5 generated by plasmin-mediated proteolysis. Proc Natl Acad Sci U S A 1999:96:5728-33.

24 O'Reilly MS, Holmgren L, Shing Y, et al. Angiostatin: a circulating endothelial cell inhibitor that suppresses angiogenesis and tumor growth. Cold Spring Harb Symp Quant Biol 1994;59:471-82.

25 O'Reilly MS, Wiederschain D, Stetler-Stevenson WG, et al. Regulation of angiostatin production by matrix metalloproteinase- 2 in a model of concomitant resistance. J Biol Chem 1999;274:29568-71.

26 Boehm-Viswanathan T. Is angiogenesis inhibition the Holy Grail of cancer therapy? Curr Opin Oncol 2000;12:89-94.

27 Boehm T, Pirie-Shepherd S, Trinh LB, et al. Disruption of the KEXI gene in Pichia pastoris allows expression of full-length murine and human endostatin. Yeast 1999;15:563-72.

28 Rowe PM. What is all the hullabaloo about endostatin? Lancet 1999:353:732.

29 Schmitz V, Wang L, Barajas $M$, et al. A novel strategy for the generation of angiostatic kringle regions from a precursor derived from plasminogen. Gene Ther 2002;9:1600-6. 